\title{
Deployment of Multimodal Services: an Ontology Driven Architecture
}

\author{
Fabio Pittarello, Augusto Celentano \\ Dipartimento di Informatica, Università Ca' Foscari di Venezia \\ Via Torino 155, 30172 Mestre (VE), Italy \\ \{pitt,auce\}@dsi.unive.it
}

\begin{abstract}
The relations between a physical environment and the services deployed in it are, in a large spectrum of cases, defined by the functional aspects of the environment, coming from its role and use in the human society. Examples of such environments are hospitals, schools, administration offices, museums, department stores, etc.; users can effectively interact with the available services if services are deployed in proper locations and accessible with interaction modalities close to users' expectation.

In an ideal design situation, all the components of a service populated environment should be defined and configured with synergy, designing at the same time the physical infrastructure and the services. In real cases, the designer of a complex interactive application faces a number of constraints due to the pre-existing infrastructure, which affects the deployment of services, hence the user interaction capabilities.

We discuss a deployment methodology and architecture based on a semantic description of environments and services, expressed by a hierarchy of ontologies. The ontologies describe the relations between the user activity, the services and the functional properties of the environment places.
\end{abstract}

\section{INTRODUCTION}

Context awareness and pervasive computing are the two main corners of a set of methodologies and technologies for designing distributed interactive applications adaptable to the user and to the environment.

A context-aware application is able to adapt services, information content and presentation to suit the user situation, depending on location, time, device, user profile, etc.. In a pervasive computing environment computing and interaction resources follow the user in a continuous environment that seamlessly allow him/her to send and receive information and execute services. The integration between the two technologies is an environment helping the user to get information through portable and ubiquitous devices in less time and with less effort [1]-[3].

Extending the context definition to include the morphological and functional features of an environment leads to the concept of ambient awareness. An ambient-aware application is able to deliver services and information in different physical environments, adapting them according to the peculiarities of the environment structure, to its functional properties and to its role and use in the human society. Environments like hospitals, schools, administration offices, museums, malls, congress halls, etc., are defined by physical and by functional properties that constrain the delivery of services. For example, an information service guiding a user to find a specific place could be delivered by catching audio/video messages in a mall, by standard signs in hospitals and schools, by human guides in a museum, by employees checking the user needs and authorization in an administration office, by a wireless information system in a congress hall during a scientific event. As shown by these examples, services offered by ambient aware applications are multimodal in nature, and can be delivered through human (guides), physical (signs) and telematic (wireless delivery) providers.

An ambient-aware application is composed of pervasive services distributed in the physical environment, accessible from local appliances, or within a wide area, or through mobile devices, delivering information and tangible objects, with which users interact in ways tailored to their context and to the properties, role and goal of the environment.

Adaptation goes beyond the knowledge of the user local situation, and relates to the functional and physical properties of the environment navigated by the user. In most cases they are high-level properties (functional or social features) that can't automatically be captured by sensors. The combined geometric and semantic description of real environments is a promising research fields that can considerably help to improve the design of interactive distributed applications [4], [5].

In this paper we discuss a methodology and an architecture for deploying services in a physical environment. The approach is based on a semantic description of environments and services, expressed by a hierarchy of ontologies. The ontologies describe the relations between the user activity, the services and the functional properties of the environment places.

The paper is organized as follows. After reviewing the related work in Section II, in Section III we discuss the relations between environment and interaction design. Section IV defines some basic concepts that are in the ontology driven architecture presented in Section V. A case study is discussed in Section VI. Section VII draws the conclusions.

\section{RELATED WORK}

Adaptation of information and services to the user context has been largely investigated. The works cited at the beginning of the previous section are a small sample of the many contributions to the field, showing a progressive shift from location awareness to more complete and sophisticated context 
descriptions. In recent years a number of proposals rely on ontological descriptions for defining the context properties in pervasive systems [6]. Ontologies have been used to describe context properties [7], different types of actors in domain specific applications [8], separate levels of context related to the user and to the domain [9]. Ontologies can describe the environment distinguished role in interactive cultural applications [10], moving towards ambient intelligence [11], [12].

All these proposals, however, consider the relationships between the environment and the user in specific domains, failing to devise a general framework for the integrated design of the environments, the functions to be performed inside and the services accessed by users.

A comprehensive context ontology for data tailoring in mobile applications is discussed in [13], [14]. As in our work, the proposal aims at developing a general methodology independent from specific domains. However, it focuses on the data content only and does not consider the influence of the environment context on user interaction.

Ontologies have been proposed for dealing with service description and identification in service computing. In [15], [16] users are supported in discovering and selecting proper services by an ontology based architecture, both at the abstract level (what kind of service is required to fulfill a functional requirement) and at the concrete level (what concrete service best exploits the user requests). The system shares with our approach the distinction between abstract and concrete levels, but does not face the match between the physical environment properties and the user goals.

Research on the semantic description of 3D environments and related information has also gradually developed in the last few years.

In the area of annotation of environment related information, some interesting proposals are based on the MPEG-7 standard. MPEG-7 descriptors are used to to index 3D scenes [17], to store semantic graphs related to a scene, allowing the users to shift between high-level descriptions of specific objects and the associated low-level geometry [18], and to describe the semantics of virtual worlds for navigating according to semantic queries (e.g., I am looking for a tall tree) [19]. While these works represent interesting proposals, the semantic description of the 3D world is related to the specific scene; no sceneindependent ontology is available, defining which are the valid relations among the objects in a certain domain.

The semantic web layers have been used by some researchers for proposing descriptions of the built environment. Young et al. [20] encode structural information, defining a set of classes, properties and constraints for describing buildings. While the proposal offers an interesting solution for representing the semantic feature of the built environment, the authors don't explicitly consider the relations with the 3D low-level objects that represent the geometric properties of the scene.

Pittarello et al. [5], [21] propose a different approach based on the web standards for storing the ambient description: the semantic web layers are used for defining the classes of semantic objects and their relations (such description is sceneindependent and generally is valid for a specific domain); the X3D standard [22] is used for describing the geometry of the 3D environment and its association with the higher-level semantic objects.

In the AEC (Architecture, Engineering and Construction) domain international organizations, national institutions and software vendors are gradually moving from a static description of 3D geometric data to a different representation that takes into account the dynamic nature of the building process, promotes interoperability, allows to incorporate different levels of details in the description of the building's parts and allows to detect existing conflicts between the different components of the process. The inclusion of higher-level data, such as the description of the material features or the usage of the associated space, is part of this process.

The concept of Building Information Modeling (BIM) has been associated in the last few years to such more dynamic and comprehensive description of the building process. For representing BIM information a number institutions and vendors have developed different data formats to describe, exchange and share information typically used within the building. The Industry Foundation Classes (IFC) specification [23], developed and maintained by the International Alliance for Interoperability (IAI [24]), represents one of the most interesting approaches. The IFC Specification proposes an open source file format and it is endorsed by ISO.

Although interesting, according to some authors, the specification is still immature [25], very complex [20] and lacks some features, such as the ability to integrate reliably nonspatial multimedia data with the 3D geometry.

\section{ENVIRONMENT AND INTERACTION DESIGN}

In an ideal situation, the design of a distributed multimodal interactive application is the result of a cooperation among a team of designers, examining the problem from different perspectives, with different experiences and skills, and different evaluation parameters in mind.

In a minimal view, useful to approach the main issues of the design phase, the team is composed (at least) by a designer of the physical environment (an architect), a designer of the application (an information technology engineer), and a designer of the interaction (a HCI expert). Experts in other fields (e.g., an expert of building safety) help the team to explore all the facets of a complex design.

In the following discussion we shall focus on the physical environment design and on the interaction design.

The outcomes of a good design include four aspects:

- the physical environment, with respect to the relations between it and the activities needed by the application;

- the (human) activities that need to be executed in it, related to the application;

- the services that need to be deployed in the environment for executing the activities;

- the assistance provided to the user for activity execution. 


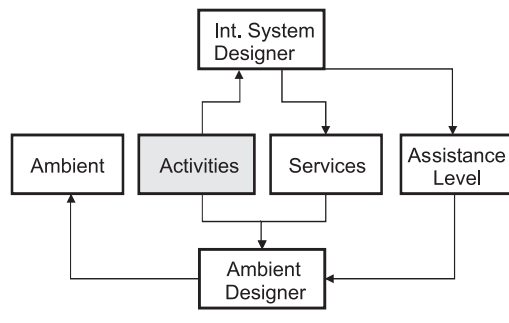

(a) Design-time optimization

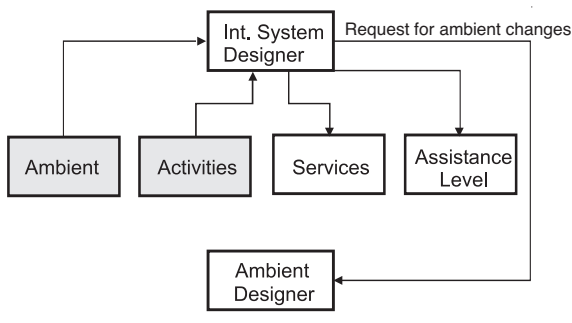

(b) Deployment-time optimization

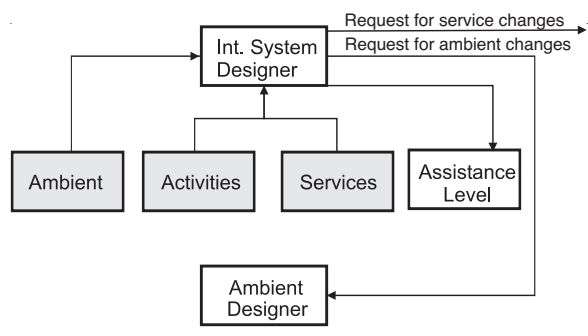

(c) Run-time optimization

Fig. 1. Scenarios for ambient and interaction design

Figure 1 illustrates three different scenarios related to different pre-existing situation at application design time. Grey nodes denote the elements already existing and no longer modifiable.

In Figure 1a the physical environment (e.g., a building, a hall, an apartment) is not yet built. The team of designers has the highest degree of freedom in designing at the same time the environment and the interaction, choosing the appliances to support the application and adapting at the same time the environment configuration, the service deployment, etc., within the constraints set by the architectural design.

In Figure $1 b$ the distributed application must be deployed in an existing physical environment, where the locations devoted to the application and the appliances for the interaction have not been chosen yet. The team of designers can collaborate to select the best deployment of the interactive services within the constraints of the physical environment.

In Figure 1c the appliances for interacting with the application are already deployed, as in a new design of an existing set of services.

We briefly comment on the three scenarios, focusing the remainder of the paper on the second one.

\section{A. Coordinated ambient and interaction design}

The case depicted in Figure 1a gives corresponds the interaction designer the highest degree of freedom in cooperating with the physical environment designer, to match at the highest level the user expectations in terms of ease and effectiveness of interaction with the application services.

Starting from the specification of the activities that different categories of users need to execute in the environment, the physical and the functional structure can be adapted to support an optimal deployment of services. The activity specifications can be given in terms of functional diagrams defining the user activities and their relations, on which the interaction designer will base the specification of embedded information services. The resulting compound document will be used by the physical environment designer as a normative specification.

\section{B. Deployment of services in an existing environment}

The case depicted in Figure $1 \mathrm{~b}$ is characterized by the presence of one or more physical environments already built. The application is defined in its overall properties, but the design of the interaction services has not been completed.
They must be designed and deployed in a way compatible with the physical constraints of the environment in which the interaction will take place. This scenario is the most frequent and only apparently without critical design aspects. Its main drawback is that the pre-existing physical environment might prevent or discourage the implementation of a number of services, or of a desirable user assistance level, because incompatible with the environment features.

The interaction designer is able to identify the activities executed in the environment, and associates them to a semantic description of the ambient. The association allows the designer to discover and mark the points of weakness of the spatial configuration in relation to the user actions. The interaction designer will then elaborate the deployment of the services according to the desired level of user assistance compatible with the environment. In critical cases, the interaction designer could ask the environment designer to foresee a change in the environment physical structure to comply with the application to be deployed. Unfortunately, in real cases, often the application is changed to fit the environment constraints.

\section{User assistance design}

In the case depicted in Figure 1c the environments and the services have already been designed and deployed. The only contribution that can be given is the design of a set of assistance services helping the user to interact at the best achievable degree of satisfaction.

As in the case above, the impossibility to reach the planned level of user assistance produces one of two outcomes: a request for changing some services, to obtain an enhancement of the information infrastructure deployment; or a more radical request for changes in the physical ambient if the integration of the actual application with more information services is not sufficient to grant the user assistance goals. This case might well happen if the environment and the application have social relevance, and mandatory regulations exist to improve the assistance to impaired people.

We shall discuss the scenario illustrated in Section III-B, presenting an architecture based on a set of ontologies describing the relations between the activities, the ambient and the services. 


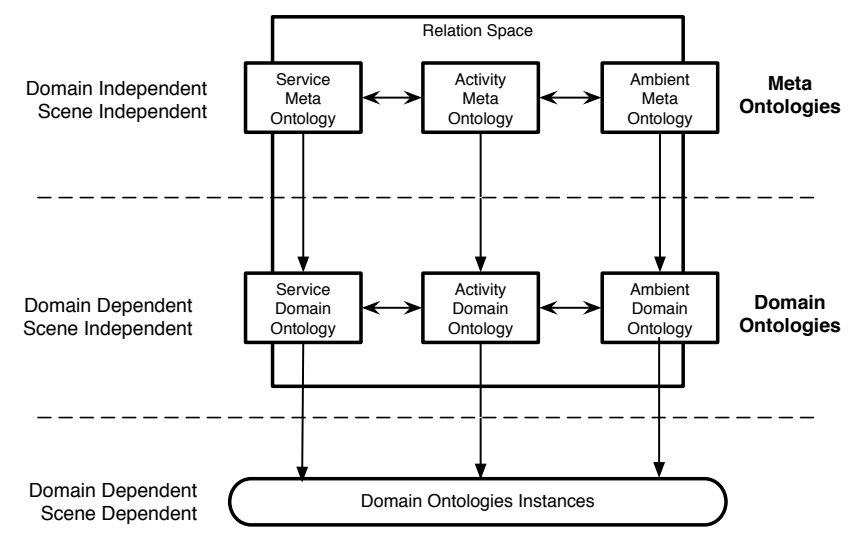

Fig. 2. The ontology and meta-ontology relations

\section{DEFINITIONS}

The association between the environment and the services is based on a conceptual description recalling a number of elementary concepts, here defined.

A service is, in a broad sense, a work executed by a provider for a consumer, producing a physical (e.g., a ticket) or abstract (e.g., a reservation) result. Services are the base components of applications. We do not elaborate further on these two concepts, nor we enter into a discussion on service computing, which is out of the scope of this paper.

A task is the execution of a single, well-specified goal by a user, according to [26], [27]. Users execute groups of coordinated and integrated tasks to perform activities. Activities are goal-directed and can be therefore distinguished in relation to the goal itself. On the other side, tasks are made of actions that represent an elementary level of operation on some device. A task may require the user move from one place to another to execute different actions. Actions may take advantage of tools (which include also service providers) that are mediators between the subject and the object of the activity.

An interaction ambient is an environment characterized by the availability of embedded communication technologies that may be accessed by a visitor in order to get information and services.

An interaction ambient is structured as a set of locations, populated by objects and artifacts, each identifying a partition in the space physically or visually delimited (e.g., by walls, windows, doors, furniture, etc.), morphologically meaningful for the user and suitable for the development of different classes of user activities. People moving through the locations can access services available in those places through the actions, which are therefore bound each to a single location.

Field services allow the user to access the service in a wide area (e.g., as provided by a Wi-Fi access point, but also by large public displays), while local services require the user to get very near or to get in touch with a specific artifact which is the terminal through which the service is delivered.

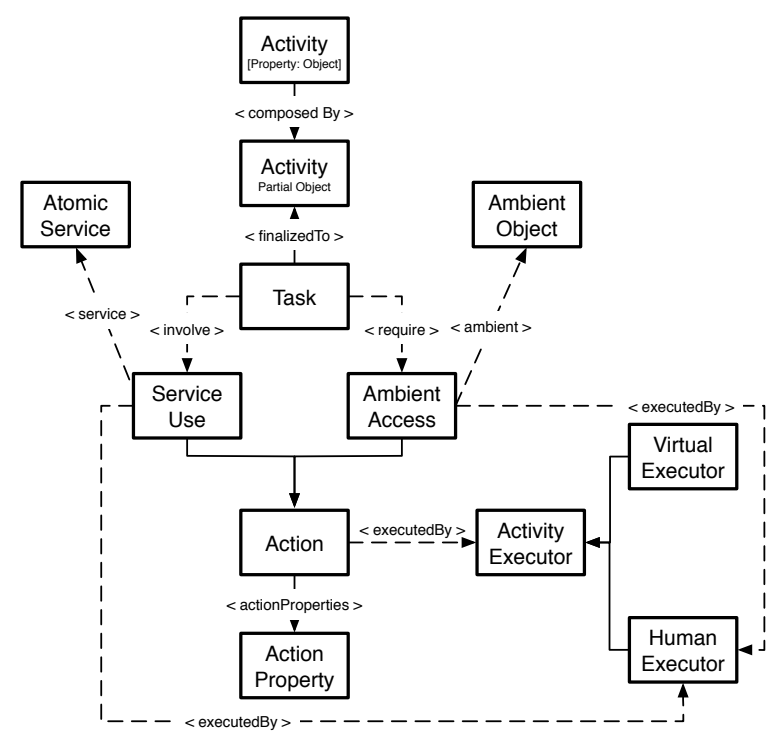

Fig. 3. The activity meta-ontology

\section{An Ontology Driven Architecture}

The software architecture described in this work is based on a set of components that cooperate to optimize the deployment of services in an existing environment where several activities take place.

Such components operate on a set of multilevel ontologies, described in [5], [28], that define the ambient, the activities performed inside of it and the available services.

An ambient ontology describes the physical environments and the semantic qualification of the objects interfacing the user with the application. A service ontology, describes the services available in the ambient and their results. An activity ontology relates services and ambients to execute in an effective way complex activities.

The ontologies are defined at three levels (Figure 2): the meta-ontology level is used for expressing high-level concepts independent from the domain and the scene of the environment, related to the definitions of service, ambient and activity; the domain ontology level declares concepts and relationships domain-dependent but still independent from the specific physical environment; the lower level is characterized by the instantiation of the concepts described at the middle level, and describes a specific case.

Due to space constraints, we cannot describe in detail the concepts and the relations that are part of all the different levels, referring the reader to [28]. A sample is shown in Figure 3, that illustrates the activity meta-ontology. Such ontology defines an activity as composed by a set of tasks according to some plan [29], [30]. To execute a task, a user needs to use a service by accessing the ambient in which the service is supplied. Both service use and ambient access are elementary actions that can be executed through a virtual executor (e.g., a program) or a human executor (e.g., an employee at a desk). In Figure 3 solid arcs represent subclass relationships, while dashed arcs denote semantic relations 


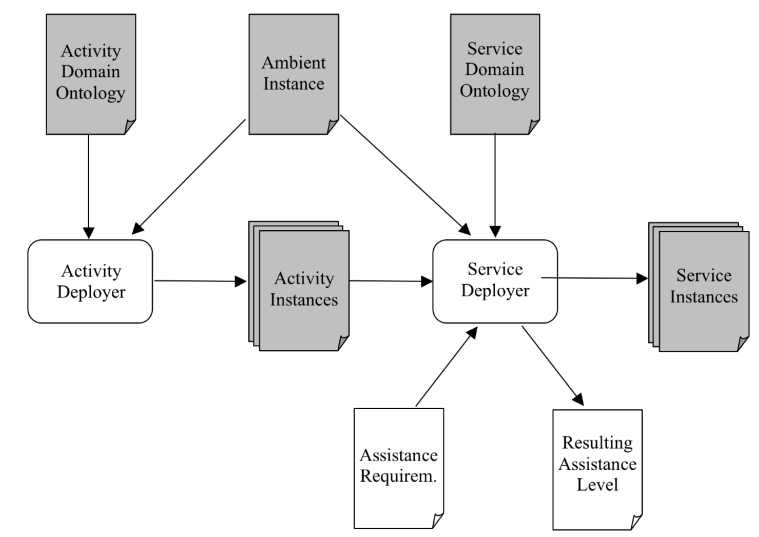

Fig. 4. The ontology driven architecture for service deployment.

identified by evocative labels.

According to the scenario described in Section III-B, part of the ontologies and the instances that derive from them are already defined: while the meta and the domain level ontologies are available for the ambient, the activities and the services, their instances are defined only if the elements they describe are already built or deployed. Such knowledge can be profitably used to generate the content of the other instances, to optimize the deployment of services.

Figure 4 shows how a set of coordinated software components can manipulate the information contained in the ontologies and in their instances, in order to obtain the description of the optimized service deployment.

The physical environment is fully described, starting from the definition of the general properties to the concrete 3D representation of its components. The activities performed inside the environment are described at the domain level and they are processed, together with the instance describing the physical ambient, by the activity deployer in order to obtain the description of the deployed activities in that specific ambient.

The activity deployer component has two different roles that vary according to the design situation:

1) The building and the activities are already defined, as in the case of a palace built and already populated with activities. The component acts as a support to define the existing instances of activities using the concepts and the relations defined at the domain level, providing an output that uses a controlled set of labels for defining the instanced actions and associated objects.

2) The building is already defined but the activities are available only at the domain level, as in the case of a reused building, where the different activities have not been assigned yet to the available locations. The component may have a more incisive role, suggesting the optimal localization for certain classes of activities. For example, it could suggest, in the context of a fine art academy, to place the atelier for painters inside the rooms that have windows pointing to the north, to benefit from a better light. Even in this case the activity deployer will provide an output that uses the set of labels
TABLE I

CONSTRAINTS FOR LOCALIZATION OF SERVICES

\begin{tabular}{|c|c|c|c|}
\hline service & req. type & strength & description \\
\hline local & user & mandatory & place next to the user path \\
\hline local & user & preferred & $\begin{array}{l}\text { place next to the start of the user } \\
\text { path }\end{array}$ \\
\hline local & user & mandatory & place along the user's line of sight \\
\hline local & user & preferred & $\begin{array}{l}\text { don't place in narrow connection } \\
\text { spaces }\end{array}$ \\
\hline local & user & preferred & place against an opaque surface \\
\hline local & user & mandatory & place next to a light source \\
\hline local & protection & preferred & place against a robust surface \\
\hline local & wiring & mandatory & $\begin{array}{l}\text { minimize distance from existing } \\
\text { wiring }\end{array}$ \\
\hline local & wiring & preferred & place next to existing partitions \\
\hline local & security & mandatory & place in controlled locations \\
\hline field & comm. & mandatory & place minimizing occlusion \\
\hline field & comm. & mandatory & $\begin{array}{l}\text { place on the ceiling/along the up- } \\
\text { per side of vertical partitions }\end{array}$ \\
\hline field & wiring & mandatory & $\begin{array}{l}\text { minimize distance from existing } \\
\text { wiring }\end{array}$ \\
\hline field & wiring & preferred & place next to existing partitions \\
\hline field & security & mandatory & place in controlled locations \\
\hline
\end{tabular}

and concept defined for the domain, applied to the real situation.

In both cases the result is a detailed description of the instance, where the different tasks that characterize a specific activity are associated to a set of (possibly ordered) locations and to an oriented path connecting them, if the accomplishment of the activities require also navigational steps.

The service domain ontology describes the different categories of services (i.e., local and field services) and their technical features (e.g., max. distance for wireless connectivity in relation to the different materials placed between the emitter and the target). All such components are processed by the service deployer that will output as a result a set of local and field services, precisely localized in the ambient and optimized according to different constraints.

Such constraints are associated to the service ontology and are related to different requirements, as shown in Table I.

Each constraint corresponds to a specific need stemming from the user, communication, protection, wiring and security requirements:

- the user requirements are focused on augmenting the perception of the availability of services and the easiness of use;

- the communication requirements are focused on maximizing the availability of a field service inside a given area;

- the protection requirements are focused on saving the local appliance and the user from physical damages;

- the wiring requirements are focused on the need to connect the service to the network; 

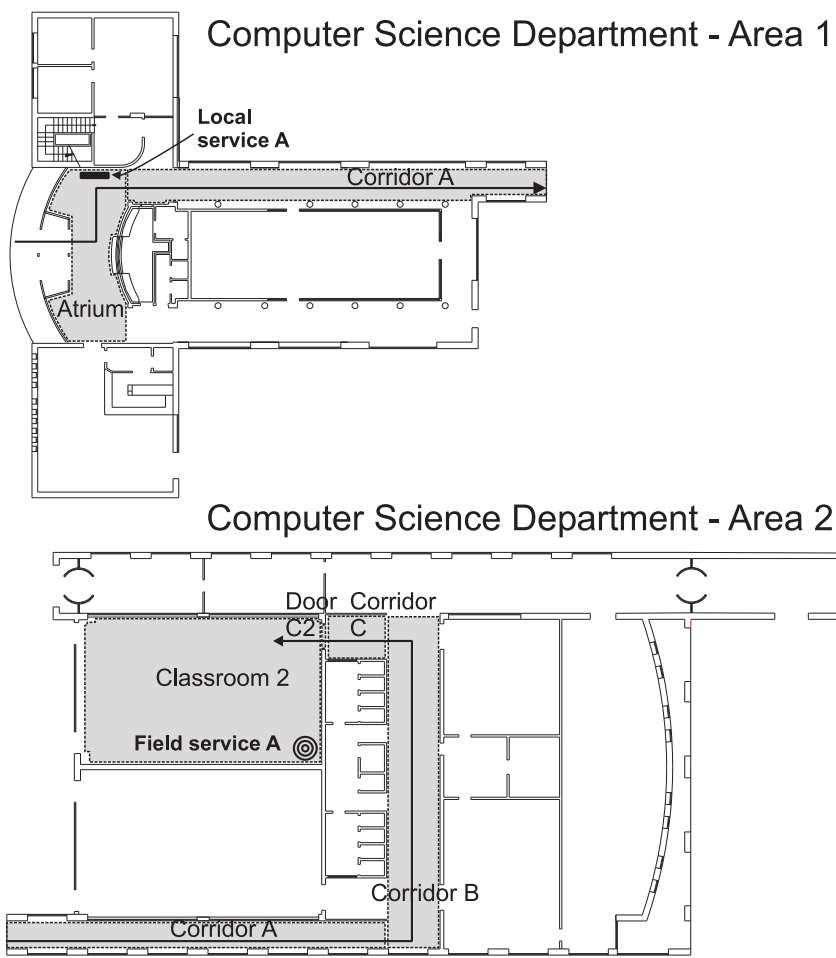

Fig. 5. The computer science Department - area 1 (entrance, services and study room) and area 2 (classes, laboratories and computer center).

- the security requirements are focused on preventing thefts of the hardware used for delivering the service.

The service deployer takes also into account the list of assistance requirements, derived from the description of the user profile and outputs, as a complementary document, the description of the resulting assistance level, which may comply or not with the initial requirements.

\section{A Case Study: The Computer Science DEPARTMENT OF CA' FOSCARI UNIVERSITY}

The building hosting the Department of Computer Science of $\mathrm{Ca}^{\prime}$ Foscari University is located in the mainland and is frequented by different user types that populate the site for executing different types of activities. While some users need to be supported mainly for their core activity (studying), occasional users, unfamiliar with the department physical structure, must be supported also for complementary tasks, such as moving inside the department to locate the relevant rooms.

Table II summarizes some properties of a few user types: the frequency of visits to the department, the availability (as a default case) of wireless personal devices, and a description of the most relevant activities executed.

This specification sets also a requirement for a specific level of assistance in accessing the services. We assume that the services have not yet been deployed, and shall do that using the architecture defined above.

The department is divided into three main functional areas connected by a set of connecting paths, but for simplicity only

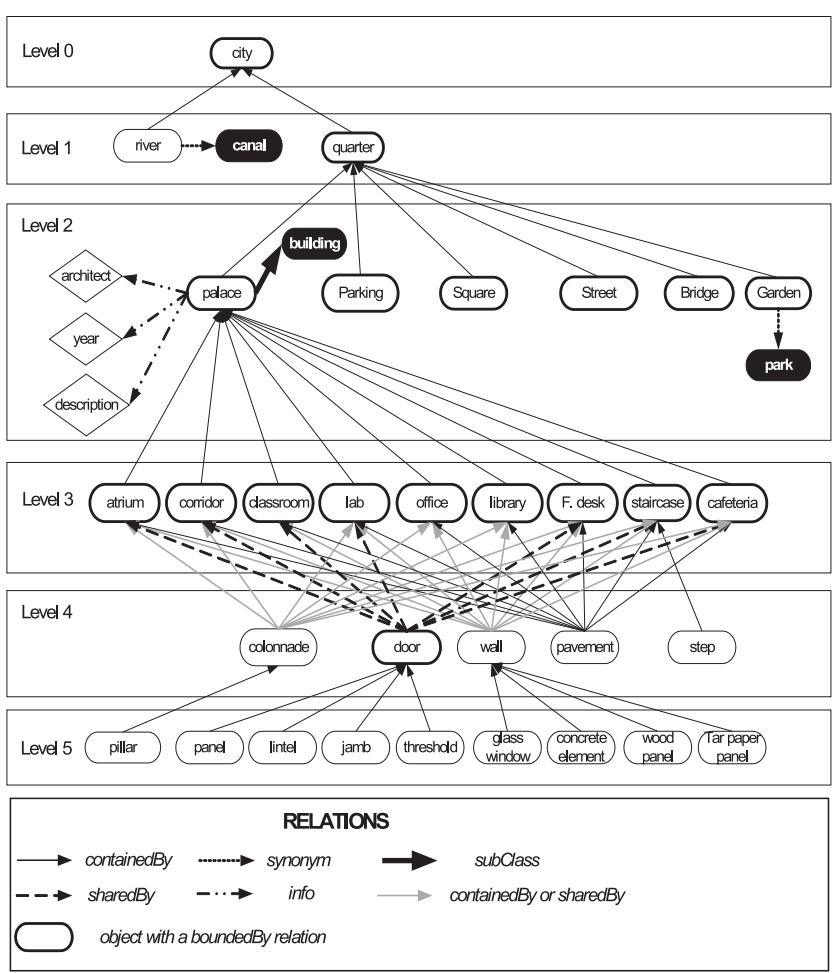

Fig. 6. The ambient domain ontology - A view centered on semantic objects and their relations.

two areas are represented in Figure 5:

- area 1 (entrance, administrative and didactic services, students study room, cafeteria);

- area 2 (classes, laboratories and computer center).

There is no clear separation of functionalities in the building; this situation doesn't help the casual visitor to find the right place.

The ambient ontology describing the building is an extension of the urban ontology defined in [21], modified to comply with this specific domain. A part of it is shown in Figure 6. The ontology shares with the general urban definition the multilevel description of objects and spaces, their coordinated definition and the classification of spaces into action spaces and connection spaces. To account for requirements about the placement of computing equipment, we extended the description of the room walls, considering the transparency and the robustness of the material. This information is used by the service deployer component as described in Section V.

To show the service deployment process, we consider a specific user type, the guest students, that are not familiar with the structure of the building, and may not be provided with a wireless device for guidance. The activity that the user has to perform in this analysis is to attend a half-day seminar in classroom 2.

Figure 5 shows the visible part of the processing done by the activity deployer: the description of the user path from the building entrance to the seminar's room and the spaces entered by the user in order to accomplish the goal. The 
TABLE II

A SAMPLE OF USER TYPES AND PROPERTIES

\begin{tabular}{|c|c|c|c|}
\hline type & freq. & w/less & tasks \\
\hline student & regular & yes & $\begin{array}{l}\text { frequenting lessons and labs, } \\
\text { studying, visiting teachers }\end{array}$ \\
\hline guest student & casual & no/yes & attending a talk \\
\hline teacher & regular & yes & teaching, doing research \\
\hline $\begin{array}{l}\text { administrative } \\
\text { staff }\end{array}$ & regular & no & doing administrative tasks \\
\hline technician & regular & yes & doing technical tasks \\
\hline accessibility & casual & no & assisting disabled people \\
\hline $\begin{array}{l}\text { assistant } \\
\text { parent }\end{array}$ & casual & no & $\begin{array}{l}\text { reaching the room for degree } \\
\text { dissertation }\end{array}$ \\
\hline emergency & casual & no & $\begin{array}{l}\text { reaching the rooms in emergency } \\
\text { situations }\end{array}$ \\
\hline
\end{tabular}

action is composed by both navigational tasks and residential tasks. Residential tasks are the core of the user action, but navigational tasks might require more support because of the user unfamiliarity with the building structure.

The system chooses to deploy a local service targeted to support the user navigation, e.g., an interactive map of the building with the description of the current activities and their localization, next to the beginning of the path; if users are not provided with wireless guidance devices and the front desk personnel is temporarily unavailable, they can get information from the map. The semantic and geometric properties of the space atrium and of the objects that compose it are analyzed by the service deployer component to find the best location, considering a minimum width for the passage of other visitors, the presence of an opaque wall for optimizing the installation, and the visibility by the users entering from the main door.

The classroom represents the end of the navigational task and the start of the core tasks, attending the seminar. The service deployer places a wireless field service in the seminar room, for complementary tasks such as consulting the teaching material; this field service may be useful also for the speaker who could access the network in the context of the talk.

While the example is focused on the deployment of communication and computing services, some criteria might be used by the service deployer to give additional useful services, e.g., providing the users with visible signs along the path, to show the right direction to follow.

\section{CONCLUSION}

Describing physical environments, activities and their relations with a formal model provided by suitable ontologies allows designers to coordinate their effort to deploy a set of pervasive services according to the environment functions. Good appliance deployment is one of the main goals in the architectural domain, often achieved mainly thanks to the designer experience. Design systems based on formal grounds could provide a set of guidelines easier to apply and verifiable in their outcome.

The use of ontologies for describing activities, services, ambients and their relations constitutes a further step towards the design of interactive systems in pervasive environments. As mobile computing spreads and the public administration regulations evolve, we can expect that a growing number of services are delivered to citizens through ICT, crossing the barrier of fixed workplaces in favor of distributed wireless access points. Existing environments could be insufficient to fulfill the needs of a really pervasive set of activities. The architecture proposed in this paper could help to find good, if not optimal, deployment guidelines for distributed services.

\section{ACKNOWLEDGMENT}

Andrea Okroglic contributed to the development of the service and activity ontologies. This work is partly supported by ACRES, Cultural Association for Economic and Scientific Research, Venice, Italy.

\section{REFERENCES}

[1] A. K. Dey, "Understanding and Using Context," Personal and Ubiquitous Computing, vol. 5, no. 1, pp. 4-7, 2001.

[2] A. Held, S. Buchholz, and A. Schill, "Modeling of context information for pervasive computing applications," in Proc. SCI2002, Orlando, FL, 2002.

[3] T. Lemlouma and N. Layaïda, "Context-aware adaptation for mobile devices," in Proc. IEEE Int. Conf. on Mobile Data Management, 2004, pp. 106-111.

[4] I. M. Bilasco, J. Gensel, M. Villanova-Oliver, and H. Martin, "An MPEG-7 framework enhancing the reuse of 3D models," in Proc. Web3D 2006, Columbia, ML, 2006, pp. 65-74.

[5] F. Pittarello and A. D. Faveri, "A semantic description of 3D environments: a proposal based on Web standards," in Proc. Web3D 2006 , Columbia, Maryland, 2006, pp. 85-95.

[6] A. Ranganathan, R. McGrath, R. Campbell, and M. Mickunas, "Use of ontologies in a pervasive computing environment," Knowl. Eng. Rev., vol. 18, no. 3, pp. 209-220, 2003.

[7] T. Strang and C. Linnhoff-Popien, "Service interoperability on context level in ubiquitous computing environments," in Intl. Conf. on Advances in Infrastructure for Electronic Business, Education, Science, Medicine, and Mobile Technologies on the Internet (SSGRR2003w), L'Aquila, Italy, 2003.

[8] H. Chen, T. Finin, and A. Joshi, "An ontology for context-aware pervasive computing environments," Knowl. Eng. Rev., vol. 18, no. 3, pp. 197-207, 2003.

[9] E.-J. Ko, H.-J. Lee, and J.-W. Lee, "An intelligent context-aware service engine based on ontology," in CIMCA '05: Proceedings of the International Conference on Computational Intelligence for Modelling, Control and Automation. Washington, DC, USA: IEEE Computer Society, 2005, pp. 485-491.

[10] M. Hatala and R. Wakkary, "Ontology-based user modeling in an augmented audio reality system for museums," User Modeling and UserAdapted Interaction, vol. 15, no. 3-4, pp. 339-380, 2005.

[11] K. Luyten, C. Vandervelpen, and K. Coninx, "Task modeling for ambient intelligent environments: design support for situated task executions," in TAMODIA '05: Proceedings of the 4th international workshop on Task models and diagrams. New York, NY, USA: ACM Press, 2005, pp. 87-94.

[12] D. Preuveneers, J. V. den Bergh, D. Wagelaar, A. Georges, P. Rigole, T. Clerckx, Y. Berbers, K. Coninx, V. Jonckers, and K. D. Bosschere, "Towards an extensible context ontology for ambient intelligence," in EUSAI, European Symposium on Ambient Intelligence, ser. LNCS. Springer, 2004, pp. 148-159.

[13] C. Curino, E. Quintarelli, and L. Tanca, "Ontology-based information tailoring," in Proc. IEEE of 22nd Int. Conf. on Data Engineering Workshops (ICDEW'06), 2006, pp. 5-5.

[14] L. Tanca, "Context-based data tailoring for mobile users," in Proc. BTW Workshops 2007, 2007, pp. 282-295. 
[15] D. Bianchini, V. D. Antonellis, and M. Melchiori, "An ontology-based architecture for service discovery and advice system,' in DEXA '05: Proceedings of the 16th International Conference on Database and Expert Systems Applications. Washington, DC, USA: IEEE Computer Society, 2005, pp. 551-556.

[16] D. Bianchini, V. D. Antonellis, M. Melchiori, and D. Salvi, "Lightweight ontology-based service discovery in mobile environments," in DEXA '06: Proceedings of the 17th International Conference on Database and Expert Systems Applications. Washington, DC, USA: IEEE Computer Society, 2006, pp. 359-364.

[17] I. M. Bilasco, J. Gensel, M. Villanova-Oliver, and H. Martin, "On indexing of 3D scenes using MPEG-7," in Proceedings of the 13th annual ACM international conference on Multimedia. ACM Press, 2005, pp. 471-474.

[18] P. Halabala, "Semantic metadata creation," in Proceedings of CESCG 2003: 7th Central European Seminar on Computer Graphics, 2003, pp. 15-25.

[19] H. Mansouri, "Using semantic descriptions for building and querying virtual environments," Ph.D. dissertation, Vrije Universiteit Brussel, 2005.

[20] M. T. Young and E. Amir, "Building knowledge about buildings," in Proceedings of the AAAI 2006 Fall Symposium on Semantic Web and Collaborative Knowledge Acquisition. AAAI Press, 2006.

[21] F. Pittarello and A. D. Faveri, "Improving access of elderly people to real environments: A semantic based approach," in Proc. of AVI 2006 , International Working Conference on Advanced Interfaces, Venezia, Italy, 2006, pp. 364-368.
[22] Web3D Consortium, "Extensible 3D (X3D) ISO/IEC 19775:2004," http://www.web3d.org/x3d/specifications/ISO-IEC-19775X3DAbstractSpecification/, 2004.

[23] "IFC Specification," http://www.iai-international.org/Model/ IFC(ifcXML)Specs.html.

[24] "International Alliance for Interoperability," http://www.iaiinternational.org/.

[25] D. A. Campbell, "Building information modeling: The web3d application for aec," in Proc. Web3D 2007. ACM Press, 2007, pp. 173-176.

[26] O. Bertelsen and S. Bodker, "Activity theory," in HCI Models, Theories, and Frameworks: Toward a Multidisciplinary Science, J. Carroll, Ed. San Francisco: Morgan Kaufmann, 2003, pp. 291-324.

[27] A. Norman, "Human-centered design considered harmful," Ambient intelligence: exploring our living environment, vol. 12, no. 4, pp. 14-19, 2005.

[28] A. Celentano, A. Okroglic, and F. Pittarello, "An ontology based approach to interaction ambient design," in Proc. Workshop on Mobile Services-oriented Architectures and Ontologies (MoSO). Mannheim, Germany: IEEE Computer Society, 2007.

[29] K. Sycara, M. Paolucci, A. Ankolekar, and N. Srinivasan, "Automated discovery, interaction and composition of semantic web services," Journ. Web Sem., vol. 1, pp. 27-46, 2003.

[30] M. Vukovic and P. Robinson, "Adaptive, planning-based, web service composition for context awareness," in Proc. Int. Conf. on Pervasive Computing, Vienna, Austria, 2004. 